\section{La Révolution française}

Cahiers de l'Institut d'histoire de la Révolution française

$20 \mid 2021$

La Révolution en 3D - Textes, images, sons

(1787-2440)

\title{
La Carmagnole de Gaston Doumergue, une period room du Musée de la Révolution française à Vizille
}

Alain Chevalier

\section{(2) OpenEdition}

Journals

Édition électronique

URL : https://journals.openedition.org//rf/5140

DOI : $10.4000 /$ Irf.5140

ISSN : 2105-2557

Éditeur

IHMC - Institut d'histoire moderne et contemporaine (UMR 8066)

Référence électronique

Alain Chevalier, "La Carmagnole de Gaston Doumergue, une period room du Musée de la Révolution française à Vizille », La Révolution française [En ligne], 20 | 2021, mis en ligne le 25 juin 2021, consulté le 28 juin 2021. URL : http://journals.openedition.org//rf/5140; DOI : https://doi.org/10.4000/Irf.5140

Ce document a été généré automatiquement le 28 juin 2021.

(c) La Révolution française 
La Carmagnole de Gaston Doumergue, une period room du Musée de la Révolution française à Vizille

\author{
Alain Chevalier
}

\title{
NOTE DE L'ÉDITEUR
}

Cet article est basé sur une communication présentée lors du colloque « La Révolution en 3D - Textes, images, sons (1787-2440) » qui s'est tenu à l'université Paris 1 PanthéonSorbonne du 14 au 16 mars 2019, organisé par le Cespra et l'IHMC-IHRF. Vous pouvez 
retrouver cette communication sur la chaîne YouTube de l'IHMC à l'adresse : https:// youtu.be/JCI17F3razY

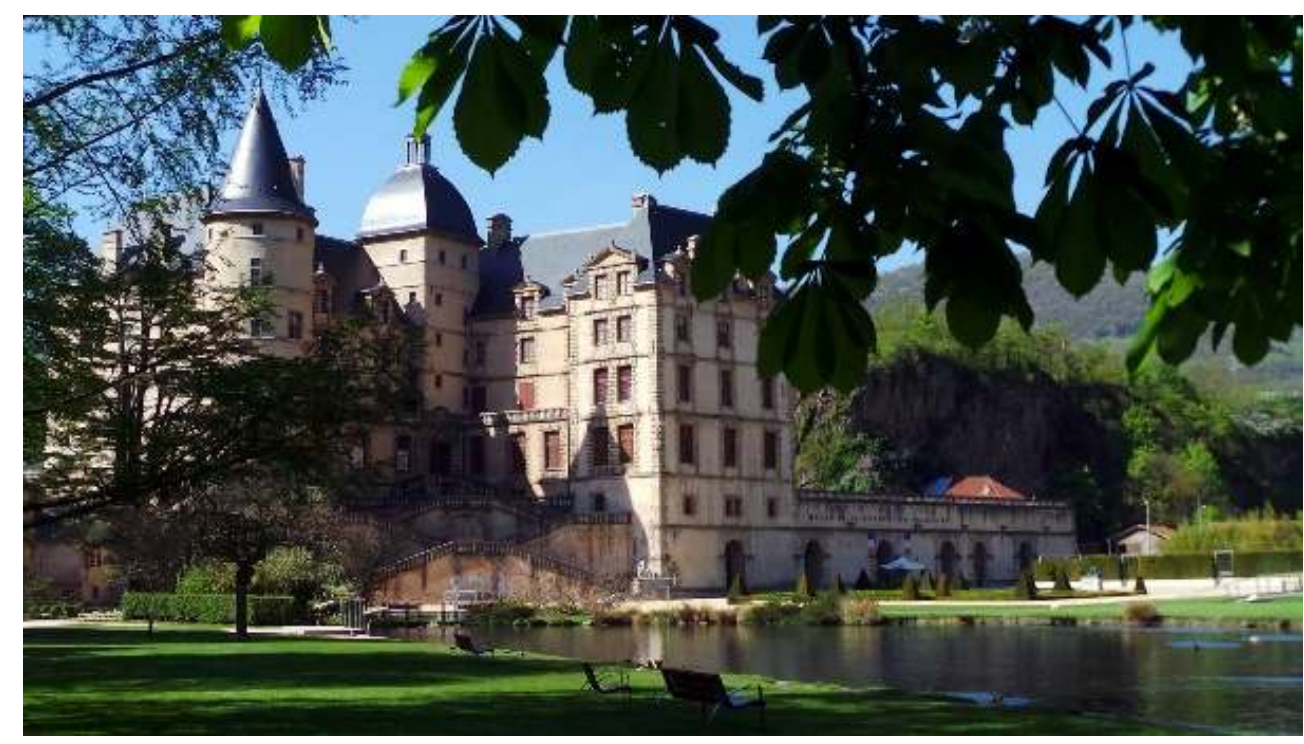

Le Musée de la Révolution française - domaine de Vizille (@ Domaine de Vizille - Département de I'Isère)

Depuis sa création en 1983 par le département de l'Isère, dans le château de Vizille où se sont tenus, le 21 juillet 1788, les États du Dauphiné qui ont réclamé la convocation des États Généraux de 1789, le musée de la Révolution française, propose une approche originale de cette période capitale de l'Histoire de France. En effet, plutôt que de suivre les voies balisées d'une narration des faits et une évocation des grandes figures de l'époque, il place la création artistique des années 1790 et celle interprétant ces mêmes années aux $\mathrm{xIX}^{\mathrm{e}}$ et $\mathrm{xx}^{\mathrm{e}}$ siècles, au cœur du processus de découverte du temps révolutionnaire et de ses conséquences. L'œuvre d'art est ainsi, à Vizille, le support de compréhension de la Révolution française, contrairement à un musée d'histoire classique, qui s'appuie avant tout sur un discours historique et soumet les objets à sa seule logique, allant parfois jusqu'au contresens. Le tableau, la sculpture, le dessin, l'estampe fournissent, sous une autre forme, autant, voire plus, d'informations et d'émotions qu'un document d'archive, encore faut-il qu'ils soient analysés rigoureusement suivant les méthodes de l'histoire et de l'histoire de l'art. Ce faisant, ces œuvres, ces images sont d'incomparables révélateurs des rapports entretenus par les contemporains et les générations suivantes, avec la rupture révolutionnaire.

Jusqu'à encore quelques décennies, les musées étaient souvent critiqués pour leur immobilisme supposé et leurs préoccupations esthétisantes prétendument surannées. Cette vision a fort heureusement changé et, aujourd'hui, l'institution muséale est souvent perçue comme un lieu d'expérimentation, devant se renouveler sans cesse pour conserver et établir un lien toujours plus fort avec ses publics, si différents par leurs attentes et leurs bagages culturels. Un des protocoles de ce laboratoire concerne les collections et leur présentation, que ce soit dans les parcours permanents ou bien dans les expositions temporaires. La muséographie est l'art même de placer les objets dans un espace avec des informations judicieusement calibrées et disposées qui instruiront le visiteur en lui procurant du plaisir. C'est ce principe même d'instruction 
et de délectation voulu par les fondateurs des musées, à l'époque de la Révolution française, qui guide le travail du conservateur du patrimoine.

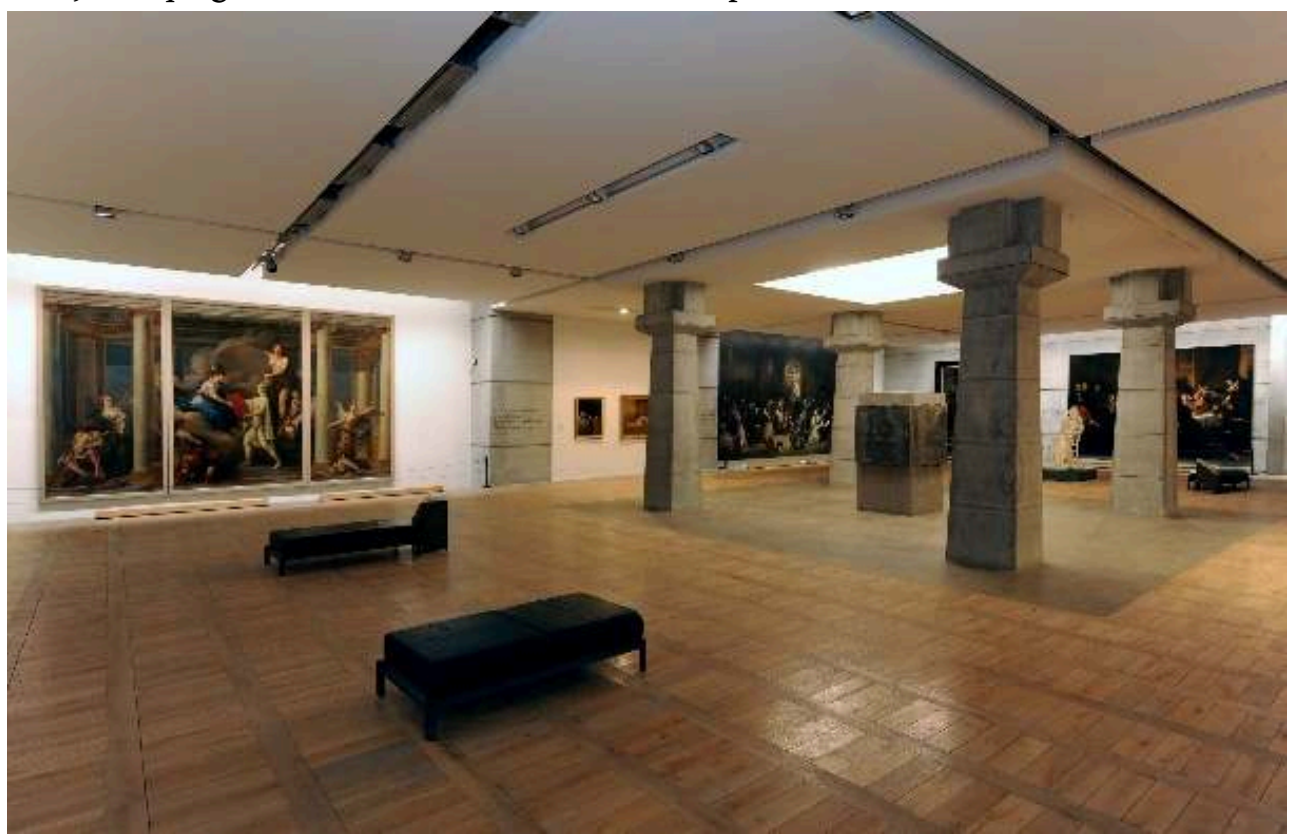

Musée de la Révolution française - Salle de la République (@ Domaine de Vizille - Département de I'Isère)

Une salle parmi toutes celles qui ont été créées au fur et à mesure de l'enrichissement des collections et de la transformation du vieux château, a souvent été commentée par les visiteurs tant elle les choque de prime abord, un effet au demeurant volontairement recherché. Le troisième niveau du musée, c'est-à-dire l'ancien étage noble que beaucoup de visiteurs continuent à venir voir en tant que tel, fut le plus difficile à rendre cohérent. La priorité, bien entendu, était la Révolution française, tout en laissant visible cependant chaque principale étape de l'histoire de l'édifice. C'est ainsi que la salle de Psyché, la bibliothèque Perier, l'escalier des Droits de l'Homme et du Citoyen, la galerie et la salle du xix ${ }^{e}$ siècle, mais également la salle du centenaire sont exclusivement consacrés à la Révolution. En contrepoint, trois salles ou groupe de petites pièces évoquent les principaux occupants du château. En premier lieu, la dynastie ducale des Lesdiguières à l'origine, au XVII siècle, du domaine de Vizille, comme exemple in loco du système féodal aboli par la Révolution ; puis la famille Perier, propriétaire de 1780 à 1895, dont les membres furent des acteurs économiques et politiques puissants qui firent entrer la vieille demeure aristocratique dans le monde de 
la haute bourgeoisie d'affaire ; enfin les présidents de la République qui, par leurs brefs séjours de 1924 à 1960, ont inscrit le château dans une mémoire populaire.

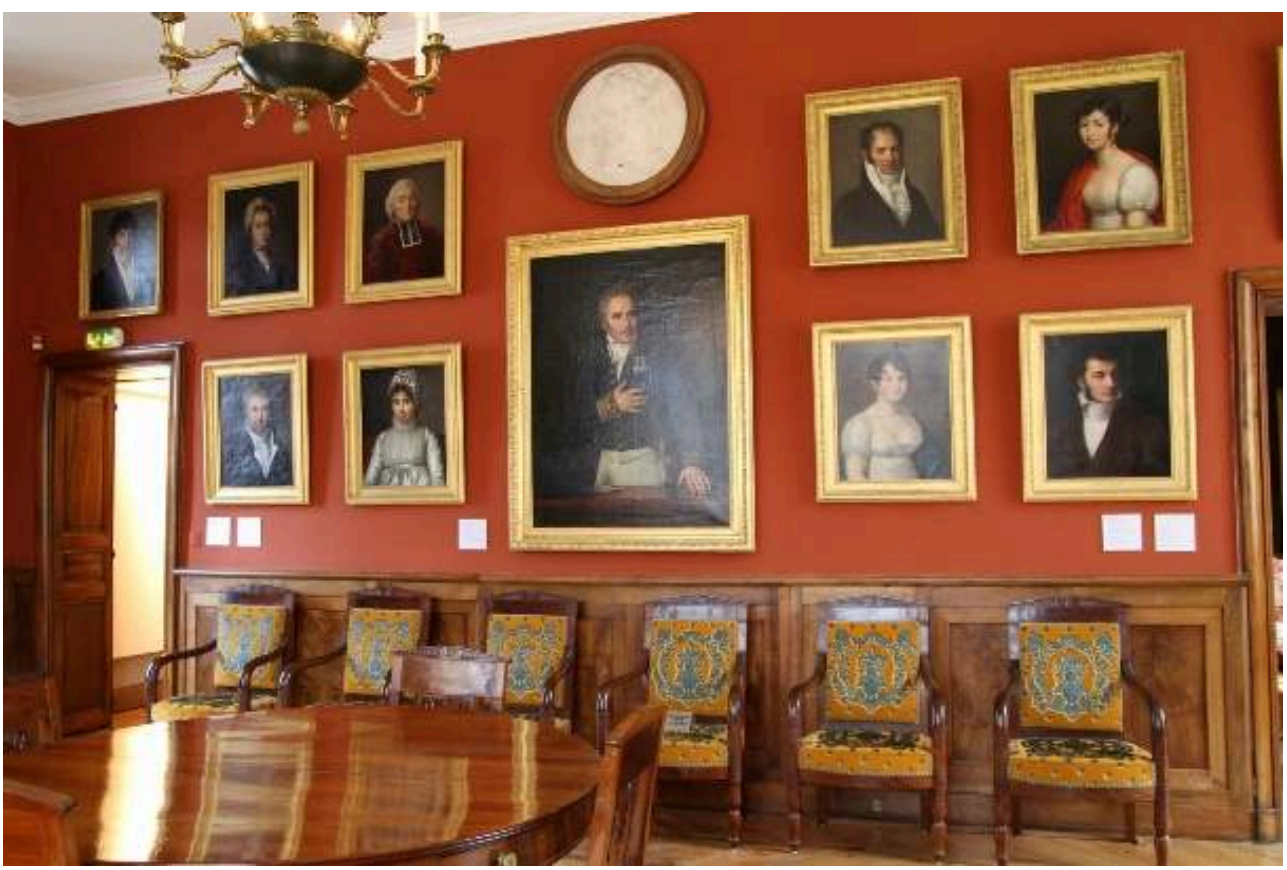

Le Musée de la Révolution française - Salle Perier (@ Domaine de Vizille - Département de l'Isère)

La salle qui nous intéresse concerne précisément la période la plus récente. Le secteur dans lequel elle est évoquée est plutôt ramassé, puisqu'il occupe seulement un vestibule ouvrant sur une pièce ronde en cul de sac, visible uniquement depuis le seuil, située dans une grosse tour médiévale d'angle donnant sur le parc' ${ }^{1}$. Il s'agissait d'une partie privative de la résidence qui permettait au chef de l'État et à son épouse, le cas échéant, de préserver leur intimité dans une petite suite aussi agréable que possible. La pièce ronde était la chambre à coucher, dont l'inconfort fut dénoncé immédiatement par Gaston Doumergue, le premier à s'être rendu à Vizille pour prendre possession du domaine en 1925. Cela étant, à partir de ce premier séjour, la chambre ronde demeura celle des présidents, au détriment de la grande chambre peu éloignée de la même aile dans laquelle avait dormi Sadi Carnot alors qu'il présidait les cérémonies du centenaire de l'Assemblée de Vizille en 1888. Cette vaste pièce, baptisée autrefois « chambre SadiCarnot », donnant sur la ville par de nombreuses fenêtres et une porte-fenêtre, avait sans doute le désagrément d'être trop exposée ${ }^{2}$. Pour autant, les deux chambres étaient meublées au départ avec des lits à baldaquin et un mobilier dans le style Louis XIII qui dataient des Casimir-Perier sous le Second Empire.

5 Les plaintes de Gaston Doumergue ayant été entendues, l'architecte des Monuments historiques Charles Halley, puisant son inspiration parmi les modèles du fameux Salon des arts décoratifs de 1925, introduisit dans le vénérable édifice, de 1926 à 1928, un vent de modernité, essentiellement par deux grands aménagements : la salle de billard et le bureau du secrétaire général de la présidence. La salle de billard au niveau deux, l'actuelle salle Charles Halley, du nom de son créateur en 1927, est, par son volume, ses boiseries et son éclairage, une salle typique de l'époque, mettant en valeur le mobilier conçu pour la résidence présidentielle et, surtout, les commandes artistiques en rapport direct avec la Révolution française dans l'Entre-deux-guerres. Le bureau du secrétariat de la présidence de la République, l'actuelle salle du XIX ${ }^{\mathrm{e}}$ siècle, a été en 
revanche dépouillée de son tissu mural d'origine, Jets d'eaux (Brunet-Meunié, d'après Edouard Benedictus, 1925), pour protéger les lés les moins endommagés. Les fragments en meilleur état ont été installés en 1998 dans l'ancienne chambre présidentielle désaffectée, qui ne présentait qu'un banal revêtement mural en tissu. De ce déménagement de tentures est née l'idée de remeubler cette pièce, non pas en tant que chambre, mais tel un salon, avec des fauteuils et un canapé de la fin du XIX siècle qui, revêtus d'un nouveau tissu vert, servaient dans la salle de billard depuis 1927, ainsi qu'un tapis de moquette de laine dont on ignore la destination d'origine ${ }^{3}$. L'éclairage de la salle est assuré par des appliques, copies de celles de la salle de billard conçues par Halley, et des lampes sur pied, dont l'intérieur du château avait été généreusement pourvu à la même époque. Un buste de Gaston Doumergue en bronze, daté de $1930^{4}$, opportunément déposé par le musée des Beaux-arts de Nîmes en 1997, placé sur la cheminée, vint compléter le tout, puisque c'est sous son septennat que les aménagements principaux avaient été réalisés, quand bien même il ne revint jamais à Vizille après 1925. Cette première étape muséographique permit au public, avec l'aménagement du vestibule présentant commentaires, photographies, films d'actualité et une chronologie complète des présidents de la République indiquant ceux qui l'honorèrent de leur présence, de disposer de solides informations sur la réalité des séjours présidentiels, dont le souvenir était resté jusqu'alors très confus ${ }^{5}$.

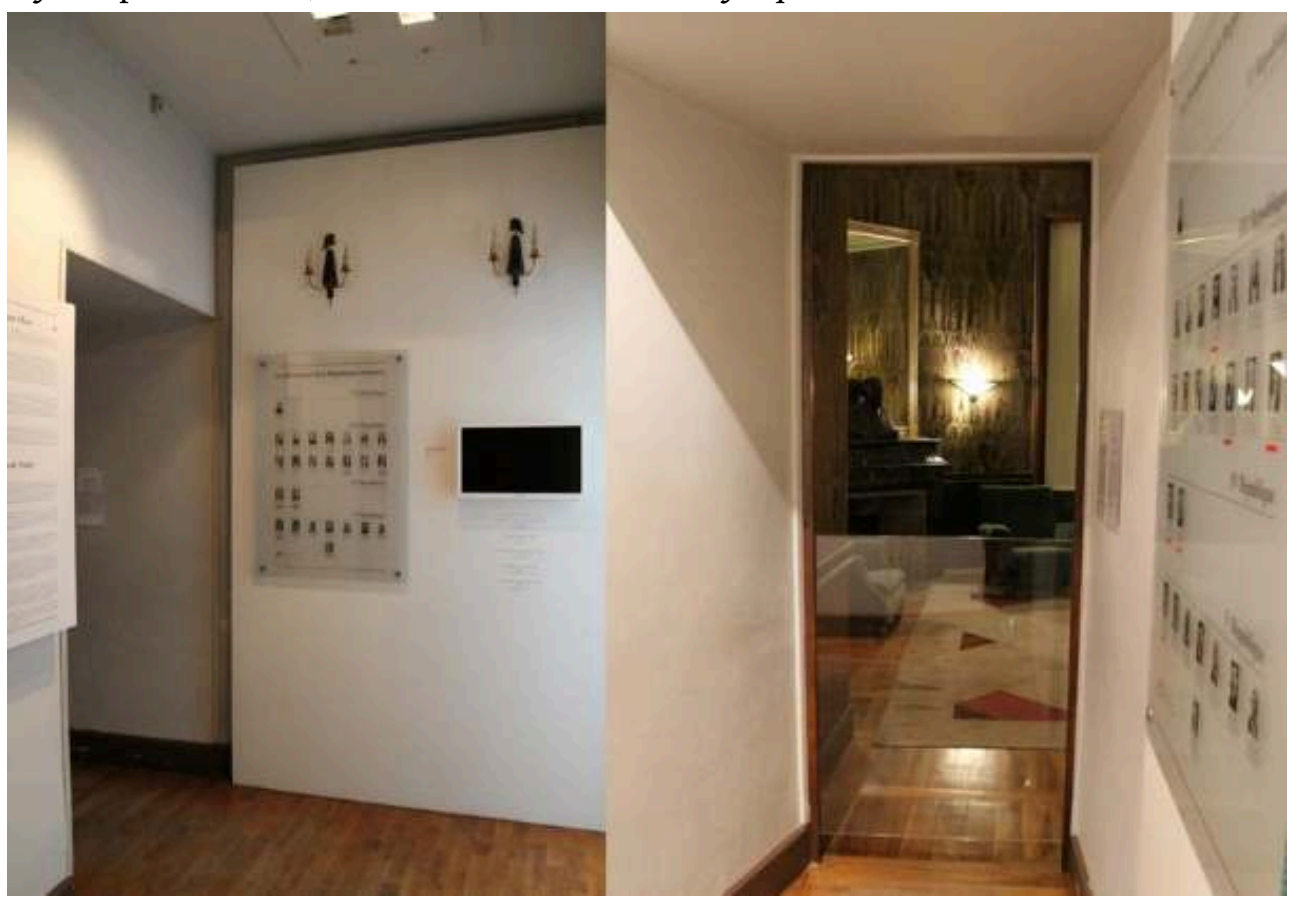

Le Musée de la Révolution française - Vestibule des présidents (@ Domaine de Vizille - Département de l'Isère)

Il manquait cependant quelque chose à cette pièce un peu compassée, aux volets toujours clos pour préserver le tissu mural de la lumière naturelle. Certes, la présence républicaine était bien expliquée, mais l'enjeu réel de l'acquisition de Vizille par l'État n'était pas directement perceptible par le visiteur. Disposer d'une nouvelle résidence estivale dans les Alpes n'était bien évidemment pas l'objet de la démarche de l'exécutif. En effet, la mise en vente du Domaine de Vizille en 1923 par son précédent propriétaire, l'industriel turinois Alberto Marone, pouvait avoir des conséquences patrimoniales désastreuses en regard des projets de démembrement et de lotissement du parc qui se 
profilaient. Le sort incertain du château, le plus important de l'ancien Dauphiné puis du département de l'Isère, dans le contexte économique défavorable de l'après-guerre, suscita néanmoins un élan national pour le sauver. Relayé au plus haut niveau, porté par le Cartel des gauches qui dirigeait alors le pays, le projet d'acquisition par l'État fit son chemin. Une loi votée au printemps 1924 précéda la signature de l'acte de vente du domaine à la fin de l'été suivant.

7 Plusieurs raisons ont sans doute conduit à cette décision. Il est possible de citer l'engagement du pouvoir centralisé dans une région alors en plein essor économique grâce à l'énergie hydraulique et à l'industrie chimique repliée depuis la guerre dans la vallée de la Romanche, ou bien l'intérêt patrimonial vis-à-vis d'un édifice aussi important, classé depuis 1862, et bien entendu le symbole républicain que représentait le château de Vizille en tant que «berceau » de la Révolution française. Cette dernière raison semble avoir compté plus que toute autre. Il fut d'ailleurs déjà question à cette époque de la création d'un musée consacré à la Révolution française, dont les soutiens ne furent hélas pas suffisants. Quand bien même, dans une période où la Troisième République subissait de plein fouet, après la victoire de 1918, une crise sociale, politique et économique que les opposants de tous bords exploitaient sans relâche, la démocratie se préoccupait sérieusement de restaurer, dans l'opinion publique française, ses valeurs fondatrices.

8 Le souvenir d'une sculpture surprenante vue en réserve, au hasard d'une visite en 1995 du château-musée de Saumur en compagnie de sa conservatrice Jacqueline Mongellaz, qui avait été en poste auparavant à Vizille, permit de trouver la solution muséographique qui devait le mieux exprimer la lutte idéologique de l'époque entre les images positives et négatives de la Révolution française. La Carmagnole (3 septembre 1792), de Charles Richefeu (1868-1945) exposée au Salon de 1923, renvoie à un épisode fameux des massacres de septembre 1792, l'assassinat de Marie-Thérèse-Louise de Savoie-Carignan, princesse de Lamballe, amie intime de la reine. On y voit un sansculotte dans un état d'excitation extrême, emporté dans une danse frénétique, 
vociférant en regardant le chef de sa victime qu'il tient par les cheveux de sa main gauche, son autre main serrant la poignée du sabre grossier dont il vient de se servir.

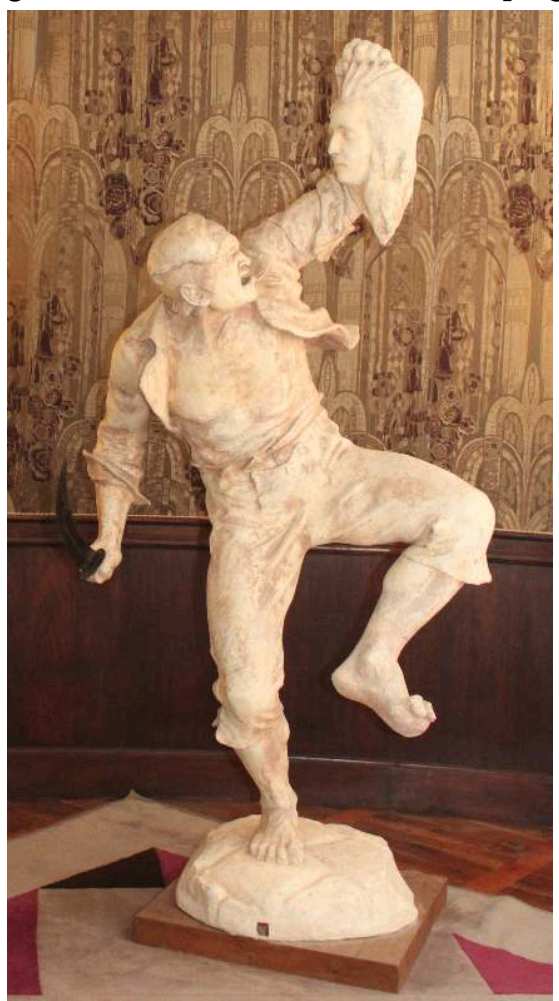

Statue La Carmagnole (@ Alexandra Lagrange, Domaine de Vizille - Département de l'Isère)

9 En considérant l'ensemble de sa production, on pourrait qualifier Richefeu de sculpteur "militariste», puisqu'une bonne partie de son œuvre concerne des soldats et des scènes militaires du ${ }^{\text {er }}$ Empire. Il est aussi surtout l'auteur, en 1918, d'un modèle de " poilu» au combat intitulé La Victoire en chantant, édité en série et placé sur des dizaines de monuments aux morts aussi bien en France qu'en Belgique. Son parcours professionnel est original. Né à Paris dans une famille bourgeoise, il commence des études de médecine qu'il abandonne pour le droit. Finalement avocat à la cour de Paris, il entre dans l'atelier du célèbre sculpteur Denys Puech un peu avant 1900 et modèle régulièrement jusque dans les années 1920. En 1914, il épouse Yvonne Bonnemère de Chavigny, issue d'une vieille famille d'avocats des environs de Saumur et dont le grandpère, Joseph-Toussaint Bonnemère, député en 1791 à l'Assemblée législative ${ }^{6}$, s'indignait de la tournure que prenait la Révolution. Richefeu, par son éducation, sa belle-famille, ses relations, est de toute évidence un conservateur et un contrerévolutionnaire convaincu. Dans sa Carmagnole réaliste et effrayante, il dénonce les violences bien réelles de la Révolution, mais d'une façon si caricaturale qu'elle traduit une haine viscérale de tout ce qui a trait à un moment essentiel et fondateur de l'histoire de France. Ces courants hostiles contre-révolutionnaires et antirépublicains furent toujours influents, mais prirent une ampleur accrue après la Première Guerre mondiale. L'acquisition du Domaine de Vizille est en quelque sorte la réaction officielle et spectaculaire à ces courants de pensée réactionnaires que la sculpture de Richefeu exprime si bien.

10 Le plâtre de La Carmagnole, réputé inexposable dans les salles du château de Saumur par des générations de conservateurs, surtout après sa dégradation lors des 
bombardements des ponts sur la Loire en juin 1940, trouva, avec l'accord de la Ville de Saumur dans le cadre d'un dépôt, sa place au Musée de la Révolution française. Cuvre considérée comme difficile, elle prenait à Vizille tout son sens. L'ancienne chambre à coucher des présidents de la République, transformée en salon de compagnie du temps $\mathrm{du}$ président Doumergue, semblait attendre une telle confrontation visuelle pour montrer avec évidence la portée symbolique de la sauvegarde du château par l'État.

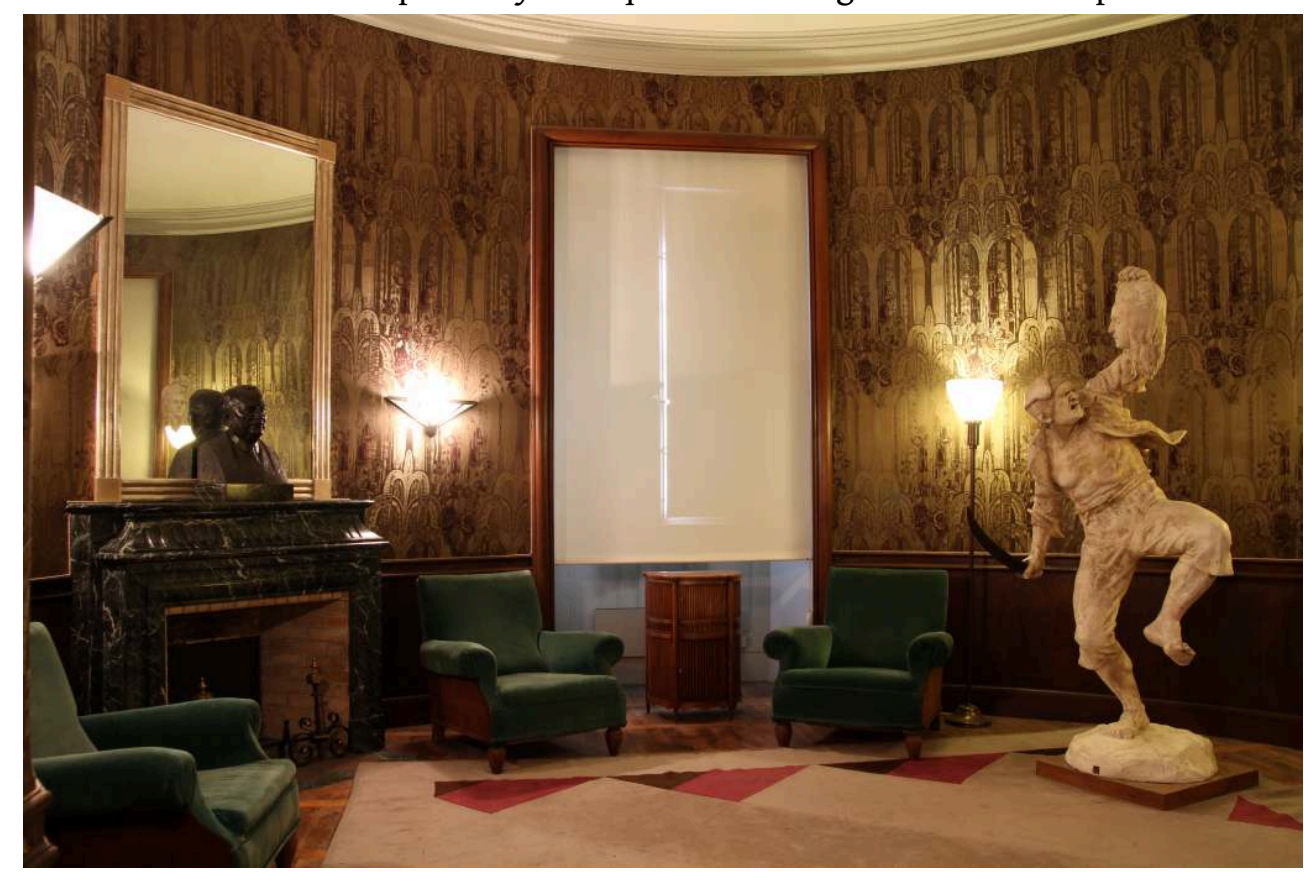

Salle Gaston Doumergue (@ Alexandra Lagrange, Domaine de Vizille - Département de l'Isère)

11 Il arrive parfois que le résultat ne soit pas à la hauteur de l'effet escompté malgré toutes les précautions préliminaires. Il suffit en effet d'une erreur d'appréciation ou bien de quelques centimètres pour affadir une mise en espace. Ce fut tout le contraire lorsque la sculpture de Richefeu arriva en 2000 après restauration. Sitôt La Carmagnole posée sur le tapis, une mécanique visuelle inattendue se mit en place grâce au grand miroir au-dessus de la cheminée ${ }^{7}$ : le visage livide de la tête décapitée, que l'on ne voit que de profil depuis le seuil de la salle, se reflète en effet frontalement comme une menace, quasiment au-dessus du visage impavide d'un Gaston Doumergue peu ému par 
l'irruption de cette créature barbare et sanguinaire qu'il affronte dignement et repousse en même temps du regard.

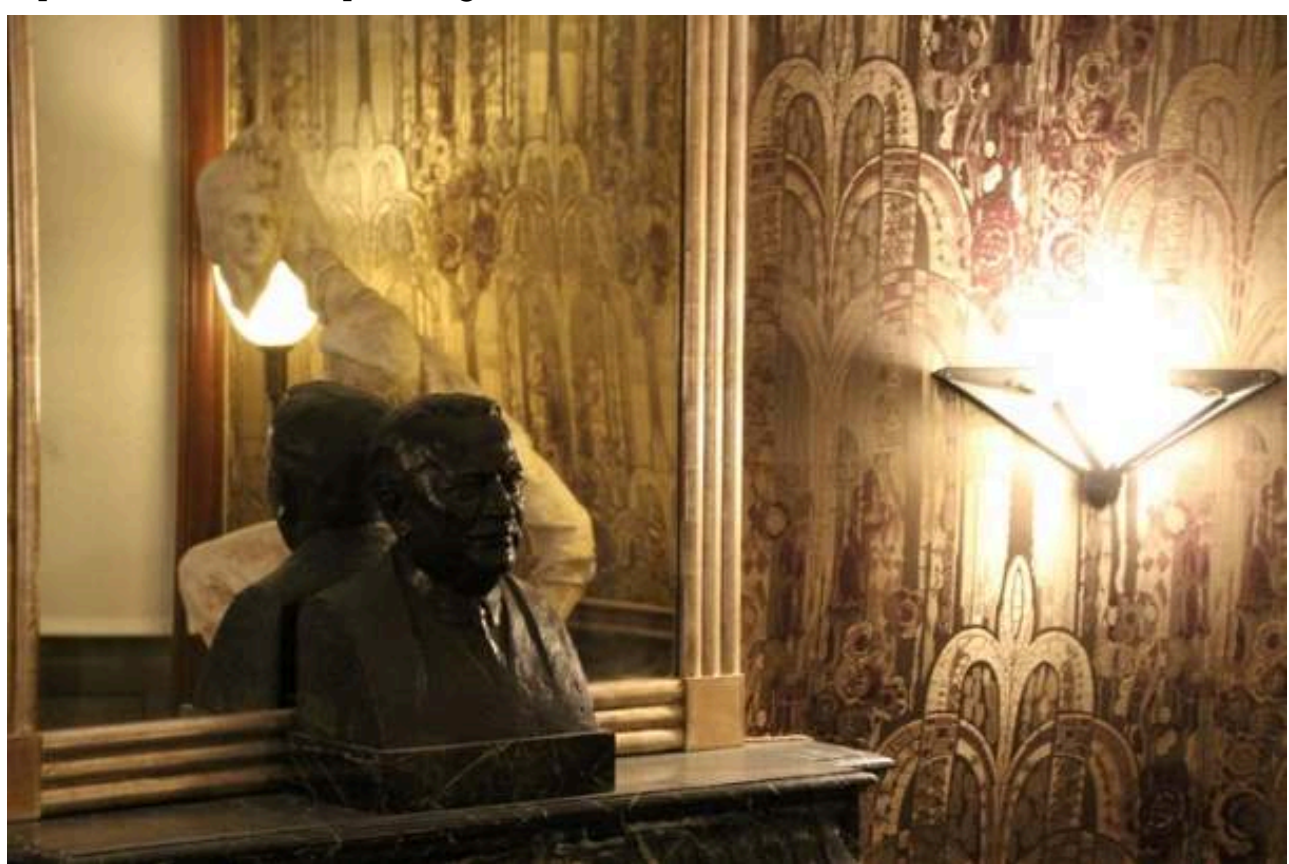

Reflet dans le miroir, salle Gaston Doumergue (@ Domaine de Vizille - Département de l'Isère)

L'effet ainsi décuplé, la confrontation de la violence révolutionnaire caricaturée à dessein avec un intérieur de la République des années 20, conduit bien évidemment le visiteur à s'interroger, passé le moment de surprise, sur le pourquoi d'une telle présentation. Un cartel placé à proximité permet de satisfaire sa curiosité, mais l'attention du spectateur et de son entourage aura été fixée durablement par l'émotion ou la surprise bien au-delà de la simple découverte visuelle de cette salle. Une enquête sur la réception du public au sortir de ce secteur du musée n'a jamais été tentée et il est généralement difficile d'évaluer précisément l'impact réel de tel ou tel dispositif, mais il est certain que les salles d'ambiance ou period room telles qu'elles ont pu être aménagées dans plusieurs espaces du musée, à chaque fois sur des modes différents, ont un pouvoir de suggestion indéniable qui captive plus facilement l'attention des visiteurs. Elles sont donc un atout essentiel des présentations de musée surtout lorsqu'il s'agit d'évoquer l'histoire par des œuvres d'art.

\section{NOTES}

1. La salle de bain adjacente, dans son état des années 1950, n'est pas accessible au public.

2. Aujourd'hui «salon de famille » des salles Perier, toujours au niveau trois.

3. Grand tapis à décor géométrique de $385 \times 360 \mathrm{~cm}$ constitué d'un assemblage de pièces de moquettes de laine de couleurs différentes.

4. CEuvre d'Henri Calvet. 
5. Voir le journal d'exposition 2002, La République dans ses murs, les Présidents au château de Vizille, 1925-1960, 2002

6. Joseph-Toussaient Bonnemère (1746-1794) est mort du typhus chez lui dans le Maine-et-Loire. 7. Le miroir à bordure en bois doré $\mathrm{du} \mathrm{xIx}^{\mathrm{e}}$ siècle, resté en place par défaut dans un premier temps, a été remplacé en 2011 par un autre aux dimensions identiques fabriqué spécialement. L'encadrement argenté se mariant avec le tissu de Benedictus s'inspire d'un modèle de Suë et Mare datant de 1921. D'une manière générale, voir pour tout ce qui concerne le décor : Un décor pour la République, le château de Vizille dans les années 1920 et 1930, Vizille, Musée de la Révolution française, 2011-12.

\section{INDEX}

Mots-clés : Musée de la Révolution française, Muséographie, Histoire de l'art, Art

Keywords: Museum of the French Revolution, Museography, Art History, Art

\section{AUTEUR}

\section{ALAIN CHEVALIER}

Musée de la Révolution française - domaine de Vizille 